\title{
氮掺杂部分石墨化碳/钴铁氧化物复合材料的制备及电化学性能
}

\author{
李甜甜赵继宽* 李尧全贞兰徐洁 \\ (生态化工国家重点实验室培育基地 (青岛科技大学) 化学与分子工程学院 青岛 266042)
}

\begin{abstract}
摘要 以甲壳胺 $(\mathrm{CTS})$ 和钴、铁盐作有机前体与反应物, 采用共沉淀法制备了 $\mathrm{CTS} /$ /钴铁层状双金属氢氧化物复合物. 样 品经过氩气氛、空气氛㷽烧, 生成氮掺杂部分石墨化碳/钻铁氧化物复合材料(N-PGC/CoFe-TMOs). CTS 热解且被过渡 金属催化生成部分石墨化碳, 同时原位氮掺杂, 氮/碳原子比例约为 1/18. N-PGC/CoFe-TMOs 具有大孔和介孔结构, 用 作超级电容器电极材料兼有双电层电容与赝电容特征. 在 $2 \mathrm{~A} \cdot \mathrm{g}^{-1}$ 电流密度下, 复合物比电容达到 $671.1 \mathrm{~F} \cdot \mathrm{g}^{-1}$, 远大于 纯钴铁氧化物比电容 $283.3 \mathrm{~F} \cdot \mathrm{g}^{-1}$, 复合物具有典型的协同效应. 电流密度增加到 $10 \mathrm{~A}^{\circ} \mathrm{g}^{-1}$ 时, $\mathrm{N}-\mathrm{PGC} / \mathrm{CoFe}-\mathrm{TMOs}$ 比电 容为 $573.3 \mathrm{~F}^{\circ} \mathrm{g}^{-1}$, 经过 5000 次充放电循环，复合物比电容保留率为 $66.4 \%$. 制备方法简便、通用，煅烧过程可一步制备 氮掺杂的部分石墨化碳并与过渡金属氧化物复合, 产物电化学性能优异.
\end{abstract}

关键词甲壳胺; 氮掺杂; 部分石墨化碳; 过渡金属氧化物; 超级电容器

\section{Synthesis and Electrochemical Properties of Nitrogen-Doped Partially Graphitized Carbon/Cobalt Iron Oxides Composite}

\author{
Li, Tiantian Zhao, Jikuan* Li, Yao Quan, Zhenlan Xu, Jie \\ (State Key Laboratory Base of Eco-Chemical Engineering (Qingdao University of Science and Technology), \\ College of Chemistry and Molecular Engineering, Qingdao 266042)
}

\begin{abstract}
With the renewable biopolymer chitosan (CTS) as a structure directing agent and organic precursor, facile coprecipitation method was applied for the cobalt and iron nitrates in solution to prepare CTS/cobalt iron layered double hydroxides composite. The LDHs sample was calcinated in a tubular furnace under Ar atmosphere via heating ramps of $5{ }^{\circ} \mathrm{C}$. $\min ^{-1}$ from room temperature to $200{ }^{\circ} \mathrm{C}$ and kept for $1 \mathrm{~h}$, then heated to $600{ }^{\circ} \mathrm{C}$ and remained for $2 \mathrm{~h}$. After the sample was cooled naturally to room temperature, it was heated again to $250{ }^{\circ} \mathrm{C}$ under air atmosphere and kept for $12 \mathrm{~h}$ to oxidize the transition metal elements. As a result, nitrogen-doped partially graphitized carbon/cobalt iron transition metal oxides nanocomposite (N-PGC/CoFe-TMOs) was obtained. X-ray diffraction, Raman spectroscopy, $\mathrm{N}_{2}$ adsorption-desorption analysis, scanning electron microscopy, high resolution transmission electron microscopy, energy-dispersive X-ray spectroscopy were carried out to characterize the structure, morphology and elemental composition of the product. Cyclic voltammetry and galvanostatic charge-discharge measurements were conducted to evaluate the electrochemical properties of N-PGC/CoFeTMOs. Experimental results showed that the CTS precursor was converted into partially graphitized carbon by pyrolysis with the help of catalysis graphitization action of transition metal elements. At the same time, the derived carbon material was successfully doped with nitrogen in situ and the N/C atomic ratio was about 1/18. N-PGC/CoFe-TMOs possessed bimodal porous texture including macropores and mesopores, exhibited combined characters of electrical double-layer supercapacitor and pseudocapacitor when used as supercapacitor electrode material. At the current density of $2 \mathrm{~A} \cdot \mathrm{g}^{-1}, \mathrm{~N}-\mathrm{PGC} / \mathrm{CoFe}-\mathrm{TMOs}$

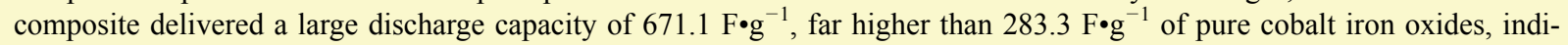
cating the typical synergistic effect between nitrogen-doped partially graphitized carbon and transition metal oxides. Even at the high current density of $10 \mathrm{~A} \cdot \mathrm{g}^{-1}, \mathrm{~N}-\mathrm{PGC} / \mathrm{CoFe}-\mathrm{TMO}$ composite still remained a specific capacity of $573.3 \mathrm{~F} \cdot \mathrm{g}^{-1}$. After 5000 charge-discharge cycles at $10 \mathrm{~A} \cdot \mathrm{g}^{-1}$, the capacitance retention was $66.4 \%$. The reported synthesis method in this work is simple and universal, and calcination process combines the nitrogen-doping, partially graphitized carbon formation with redox-active transition metal oxides synthesis in one step, endowing the product with excellent electrochemical properties.

Keywords chitosan; nitrogen doping; partially graphitized carbon; transition metal oxides; supercapacitor
\end{abstract}

\section{1 引言}

超级电容器( supercapacitor, SC) 是一种新型储能器
件, 具有功率密度大、充放电快速、循环寿命长等优 点 $^{[1]}$. 电极材料是超级电容器的重要组成部分, 对器件 性能有重要影响. SC 电极材料包括碳材料、金属氧化

\footnotetext{
*E-mail: forestzhao@163.com

Received January 10, 2017; published April 23, 2017.
}

Supporting information for this article is available free of charge via the Internet at http://sioc-journal.cn.

Project supported by the National Natural Science Foundation of China (No. 21403121), the Natural Science Foundation of Shandong Province of China (No. ZR2013BQ013) and the Project of Shandong Province Higher Educational Science and Technology (No. J14LC13).

项目受国家自然科学基金(No. 21403121)、山东省自然科学基金(No. ZR2013BQ013)和山东省高等学校科技计划(No. J14LC13)资助. 
物、金属氢氧化物、导电聚合物等 ${ }^{[2 \sim 5]}$. 各种材料都有 自身的优点与不足, 其中碳材料导电性较好但比容量较 低, 其他噟电容器电极材料氧化-还原活性高, 比电容 大, 但自身导电性较差, 循环稳定性与倍率特性限制其 应用. 调控碳纳米材料结构并与高氧化-还原活性物质 相复合是制备 SC 先进电极材料的重要途径 ${ }^{[2 ~ 8]}$.

典型碳材料包括石墨烯、碳纳米管、活性碳、介孔 碳等 ${ }^{[2,5,9]}$. 其中, 石墨烯与碳纳米管中六元环结构有利 于电子传输; 活性碳与介孔碳具有高比表面积, 但碳原 子结构有序性差, 导电性较低. 无定形碳材料经高温 (2000 ${ }^{\circ} \mathrm{C}$ 以上)石墨化处理, 结晶度与导电性增强, 但是 比表面积显著降低, 合理调控碳材料的比表面积与导电 性对于提高碳材料电化学性能有重要影响. 近年来, 研 究人员以过渡金属盐 ${ }^{[10,11]}$ 、过渡金属氢氧化物 ${ }^{[12]}$ 为催化 剂前体，通过催化石墨化作用在低温条件下 $(<$ $1000{ }^{\circ} \mathrm{C}$ )制备部分石墨化碳(partially graphitized carbon, PGC)材料, 产物的倍率特性高, 并且在水性和有机电解 质中都具有较高的能量密度和功率密度.

为进一步提高 $\mathrm{SC}$ 电极材料性能, 人们还制备了杂 原子掺杂的碳材料, 其中氮掺杂碳 ${ }^{[3,13 ~ 16]}$ 尤其引人注目. 氮原子由于原子半径较小而具有较高的电负性, 氮原子 掺杂的碳材料表面极性增强, 水性电解液更易于润湿电 极材料表面 ${ }^{[13]}$. 另外, 吡咯型氮、吡啶型氮可赋予电极 材料氧化-还原活性和赝电容特性, 石墨化型氮则有助 于提高碳材料导电性 ${ }^{[13 ~ 15]}$. 甲壳胺(CTS)作为来源广泛 的生物聚合物越来越多地用于可持续碳材料(sustainable carbon materials) ${ }^{[17]}$ 研究. 由于含有丰富的碳元素与氮元

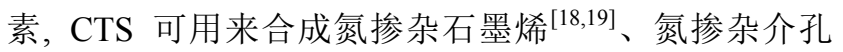
碳 ${ }^{[20,21]}$ 等材料. 氮掺杂碳作为 $\mathrm{SC}$ 电极材料具有高比电 容和良好的循环稳定性 ${ }^{[19,20]}$.

类水滑石, 又称层状双氢氧化物(Layered Double Hydroxides, LDHs), 是一类重要的层状功能材料 ${ }^{[22]}$. LDHs 作为前躯体可制备组成和结构多样的双金属氧化 物. 含过渡金属元素的类水滑石及其氧化产物, 都具有 典型的电化学活性, 已成为噟电容器电极材料的重要组 成部分 ${ }^{[22,23]}$. 为克服导电性差的不利因素, 研究人员将 LDHs 及其氧化物分别与碳纳米管、石墨烯等构建复合

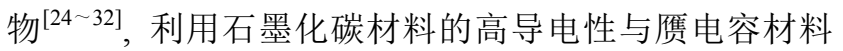
的高氧化一还原活性来提高复合电极材料电化学性能. 此外, 氮掺杂碳材料与 LDHs 及其氧化物复合也日益受 到人们重视. $\mathrm{Xu}$ 等 ${ }^{[33]}$ 以二氧化硅为模板, 以酚醛树脂和 三聚氰胺为前体, 制备了氮掺杂碳空心球, 控制勃姆石 在碳球表面多次沉积(10 次), 采用水热法成功制备了氮 掺杂碳/镍铝层状双金属氢氧化物双壳层空心微球, 产 物用作超级电容器电极材料表现出优异的电化学性能. Hao 等 $^{[34]}$ 以甲壳胺为有机前体经过热解制备了碳气溶 胶并进行活化, 经过水热反应、㷽烧处理制备了纳米针 状 $\mathrm{MnCo}_{2} \mathrm{O}_{4.5}$ 碳气溶胶杂化物, 在 $0.2 \mathrm{~A} \cdot \mathrm{g}^{-1}$ 电流密度下
比电容达到 $380 \mathrm{~F} \cdot \mathrm{g}^{-1}$, 大于相同条件下纯碳材料比电 容. 张海军等 ${ }^{[35]}$ 利用甲壳胺的成膜性能，涂制了甲壳 胺/氯化镍复合膜, 经过煅烧生成多孔碳/氧化镍复合材 料, 在 $0.1 \mathrm{~A} \cdot \mathrm{g}^{-1}$ 电流密度下比电容达到 $355 \mathrm{~F} \cdot \mathrm{g}^{-1}$, 且具 有良好的循环稳定性.

研究结果表明, 制备部分石墨化碳、氮掺杂碳并与 金属氢氧化物或氧化物复合是提高 SC 电极材料性能的 重要研究方向. 然而, 操作步骤多、耗时长不利于材料 应用，复合物比电容有待进一步提高，且少有报道兼顾 氮掺杂、部分石墨化碳结构调控以及与氧化物复合等综 合特征. 寻求简便、高效、通用的材料制备方法仍是一 项亟待解决的重要课题. 为此, 本文设计了一种制备电 极材料的简便途径，即采用甲壳胺为结构导向剂，通过 共沉淀反应制备了一系列甲壳胺/LDHs 复合物, 样品经 过耘烧，原位制备了氮掺杂部分石墨化碳/过渡金属氧 化物(N-PGC/TMOs)纳米复合材料. 对产物结构、形貌、 元素组成与电化学性能等进行表征与分析, 为开发高性 能 SC 电极材料提供科学依据.

\section{2 结果与讨论}

\section{1 结构、形貌表征与元素分析}

\subsubsection{X 射线衍射(XRD)}

分别以甲壳胺(CTS)、十二烷基苯磺酸钠(SDBS)及 其混合物为有机前体制备了 $\mathrm{CTS} / \mathrm{CoFe}-\mathrm{LDHs}$ 、SDBS/ CoFe-LDHs 和 CTS-SDBS/CoFe-LDHs 有机/无机复合材 料, 上述样品经过氩气氛炦烧后分别记为 $\mathrm{N}-\mathrm{PGC} /$ CoFe、PGC/CoFe 和 N'-PGC/CoFe, 再经过空气氛裧烧 后分别记为 $\mathrm{N}-\mathrm{PGC} / \mathrm{CoFe}-\mathrm{TMOs} 、 \mathrm{PGC} / \mathrm{CoFe}-\mathrm{TMOs}$ 和 N'-PGC/CoFe-TMOs, 并用作 SC 电极材料研究其电化学 性能. 为分析烣烧过程中目标产物的形成机制, 文中分 别测试了 CoFe-LDHs 及其复合物的 XRD 图谱(图 S1, 支持信息), $\mathrm{CoFe}-\mathrm{LDHs}$ 复合物氩气氛耘烧产物(图 1a)、 氩气氛与空气氛耘烧产物(图 1b)的 XRD 图谱.

实验结果表明, 采用共沉淀法制备的 CoFe-LDHs 及其复合物前体均具有典型的层状结构特征(图 S1), 由 于 $\mathrm{Fe}^{3+}$ 易于沉淀, 样品中还含有少量 $\mathrm{Fe}(\mathrm{OH})_{3}$ 杂质. 图 1a 显示, 有机前体/CoFe-LDHs 复合物经氩气氛裧烧后, 其中的钴、铁过渡金属元素以 $\mathrm{CoFe}$ 合金或 $\mathrm{Co}$ 单质形 式存在, 样品在衍射角为 $45.0^{\circ} 、 65.5^{\circ}$ 出现的衍射峰分别 与 CoFe (JCPDS: 65-4131)的(110)、(200)晶面衍射相对 应, 在衍射角为 $44.1^{\circ} 、 51.5^{\circ}$ 和 $76.0^{\circ}$ 出现的衍射峰则对 应于 Co (JCPDS: 15-0806)的(111)、(200)和(220)晶面衍 射. 该结果表明, 有机前体/钴铁类水滑石经过惰性气氛 煅烧，有机组分碳化，复合物中的过渡金属元素经碳热 反应 ${ }^{[36]}$ 而被还原，形成合金或金属单质.

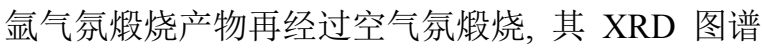
发生明显变化, 如图 $1 \mathrm{~b}$ 所示. N-PGC/CoFe-TMOs 和 N'-PGC/CoFe-TMOs 样品在 $18.5^{\circ} 、 30.9^{\circ} 、 36.5^{\circ} 、 44.7^{\circ}$ 、 
$58.9^{\circ}$ 和 $64.8^{\circ}$ 出现衍射峰, 分别与标准图谱 $\mathrm{Co}_{3} \mathrm{O}_{4}$ (JCPDS: 43-1003)、 $\mathrm{Fe}_{3} \mathrm{O}_{4}$ (JCPDS: 65-3107)的(111)、 (220)、(311)、(400)、(511)和(440)晶面衍射相对应, 并 且衍射角均介于 $\mathrm{Co}_{3} \mathrm{O}_{4}$ 和 $\mathrm{Fe}_{3} \mathrm{O}_{4}$ 标准图谱相同晶面对应 的衍射角之间. $\mathrm{Co}^{3+} 、 \mathrm{Fe}^{3+}$ 半径数值相近, 分别为 0.610 $\AA$ 和 $0.645 \AA^{[37]}$. 钴、铁均可形成具有面心立方晶型的氧 化物 $\mathrm{Co}_{3} \mathrm{O}_{4}$ 和 $\mathrm{Fe}_{3} \mathrm{O}_{4}$, 其中 $\mathrm{Co}_{3} \mathrm{O}_{4}$ 衍射峰对应 $2 \theta$ 角度略 大于 $\mathrm{Fe}_{3} \mathrm{O}_{4}$ 相同晶面的衍射角. 以上 XRD 结果表明, N-PGC/CoFe-TMOs 和 N'-PGC/CoFe-TMOs 样品均含有 具面心立方晶型且分布均匀的钴铁双金属复合氧化物, 此二样品中的过渡金属单质经过空气氛㷽烧完全转变 为复合金属氧化物.
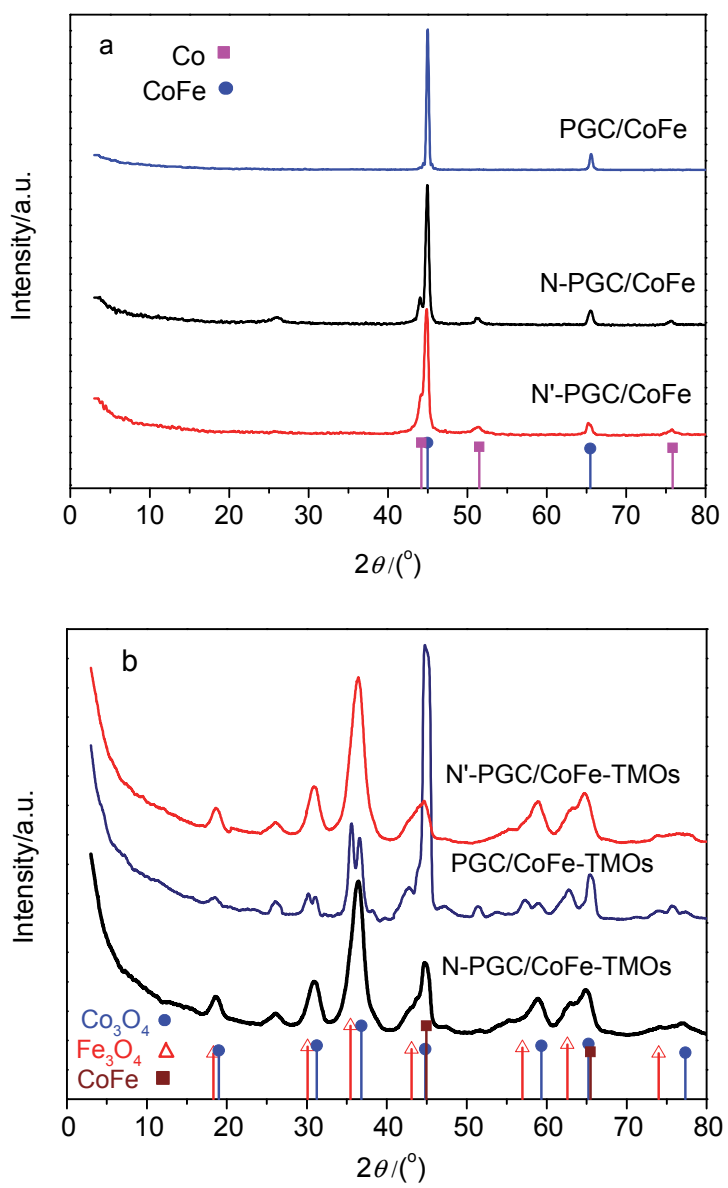

图 1 有机前体/CoFe-LDHs 氩气氛㷽烧产物(a)和后续空气氛煅烧产 物(b) XRD 图谱

Figure 1 XRD patterns of calcinated organic precursor/CoFe LDHs in Ar atmosphere (a) and followed in air atmosphere (b)

而 PGC/CoFe-TMOs 样品, 仅在 $18.5^{\circ}$ 处出现单一衍 射峰, 在上述其他衍射角附近均裂分为双衍射峰, 分别 对应于 $\mathrm{Co}_{3} \mathrm{O}_{4}$ 或 $\mathrm{Fe}_{3} \mathrm{O}_{4}$ 标准图谱(111)、(220)、(311)、 (400)、(511)和(440)晶面衍射. 两套 $X$ 射线衍射图谱共 存, 表明 PGC/CoFe-TMOs 样品中钴、铁元素单独形成 氧化物并在此基础上物理混合, 二者未形成均匀的复合 金属氧化物. 另外, PGC/CoFe-TMOs 样品在 $44.8^{\circ}$ 处存 在强衍射峰, 对应于 CoFe(JCPDS: 65-4131)(110)晶面衍
射. 按照衍射峰位置与相对峰强度大小可判断, PGC/CoFe-TMOs 样品中还含有部分未氧化的钴铁合金. 根据后续扫描电镜实验结果可知, $\mathrm{PGC} / \mathrm{CoFe}-\mathrm{TMOs}$ 样 品颗粒粒径较大 $(154 \mathrm{~nm})$, 可能不利于过渡金属在空气 氛中充分氧化. 而 N-PGC/CoFe-TMOs 样品(平均粒径 $50 \mathrm{~nm}$ ) 和 N'-PGC/CoFe-TMOs 样品(平均粒径 $80 \mathrm{~nm}$ )尺 寸较小, 在相同实验条件下经过惰性气氛与空气氛衫 烧, 其中的过渡金属可完全转化为金属氧化物. 氧化物 结构将有利于提高产物电化学性能.

从图 $1 \mathrm{~b}$ 还可以看出, N-PGC/CoFe-TMOs、PGC/ CoFe-TMOs 和 N'-PGC/CoFe-TMOs 三样品在 $2 \theta$ 为 $26.1^{\circ}$ 处均出现衍射峰, 对应于石墨化碳(002)晶面的衍射 ${ }^{[10]}$, 说明三种样品中都存在石墨化碳，充分表明过渡金属元 素对碳材料具有低温催化石墨化作用.

\subsection{2 拉曼光谱与高分辨透射电镜(HRTEM)表征}

拉曼光谱是研究碳材料及其复合物的重要实验手 段，可用于分析碳原子结构的有序性、缺陷等信息 ${ }^{[38]}$. 图 2 分别给出了 N-PGC/CoFe-TMOs、PGC/CoFe-TMOs 和 N'-PGC/CoFe-TMOs 的拉曼光谱图. 从图中可以看 出, 三样品在 1330 和 $1580 \mathrm{~cm}^{-1}$ 附近均出现拉曼峰, 分 别为 $\mathrm{D}$ 带和 $\mathrm{G}$ 带 ${ }^{[25,38]}$. 其中, $\mathrm{D}$ 带强度与石墨化碳的结 构缺陷、杂原子掺杂以及无序碳结构有关; $\mathrm{G}$ 带对应 $\mathrm{sp}^{2}$ 碳原子的 $E_{2 \mathrm{~g}}$ 模式面内振动, $\mathrm{G}$ 带强度代表着碳材料的 结晶度与有序性. 拉曼光谱测试结果表明, 制备的碳材 料同时含有有序结构碳与无定形碳. 以 CTS 为有机前 体制备的复合物 N-PGC/CoFe-TMOs 对应的 G 带峰强度 最大, 峰形对称性好, 表明碳原子六元环结构较完整, 有利于电子传输. 当 CTS 被 SDBS 部分取代、完全取代 来制备 N'-PGC/CoFe-TMOs 和 PGC/CoFe-TMOs 复合材 料时, $\mathrm{G}$ 带峰强度降低表明形成的有序结构碳较少.

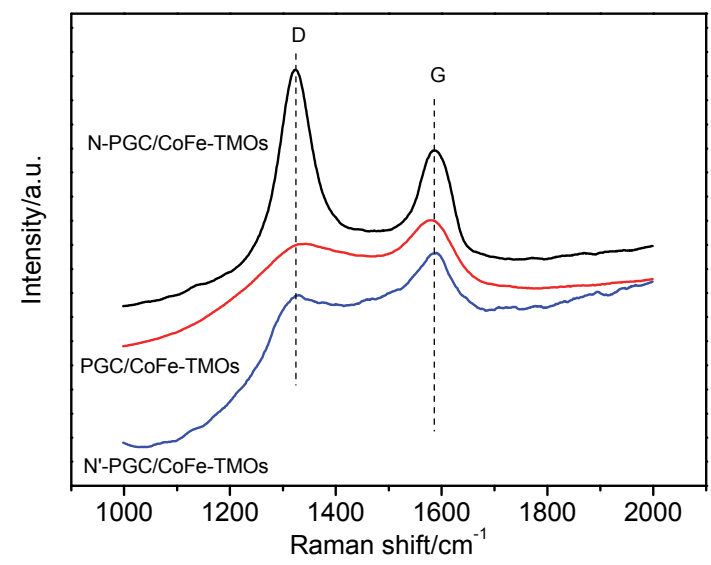

图 2 钴铁氧化物复合材料拉曼光谱

Figure 2 Raman spectra of CoFe-TMOs composites

另外, $I_{\mathrm{D}} / I_{\mathrm{G}}$ 强度比还与碳材料中缺陷密度有关 ${ }^{[30]}$, N-PGC/CoFe-TMOs、N'-PGC/CoFe-TMOs 和 PGC/CoFeTMOs 的 $I_{\mathrm{D}} / I_{\mathrm{G}}$ 强度比分别为 $1.34 、 0.80$ 和 0.89 , 甲壳胺 
前体经过炷烧生成的碳材料中结构缺陷也较多, 这可能 与碳材料中氮原子掺杂有关. 而这些氮掺杂缺陷点也是 电化学反应的活性位点 ${ }^{[13]}$, 将有利于提高复合电极材 料的电化学性能.

为进一步分析敖烧产物中碳材料的结构特征, 本文 对 N-PGC/CoFe-TMOs、N'-PGC/CoFe-TMOs 和 $\mathrm{PGC} /$ CoFe-TMOs 样品进行了高分辨透射电镜(HRTEM)观测, 碳材料的形貌特征如图 3 所示. 从图中可以看出, 制备 的碳材料中均包括层状有序结构碳(如图中箭头所指部 分)和无定形碳(如图中虚线所包围的区域). 经计算可 知, 相邻碳层之间的重复间距为 $0.37 \mathrm{~nm}$, 与文献 ${ }^{[14]}$ 报 道的氮掺杂石墨化碳材料结构类似. 对比可发现, N-PGC/CoFe-TMOs 样品(图 3a)中层状结构分布面积最 广且较均匀; N'-PGC/CoFe-TMOs 样品(图 3b)无定形碳 区域比较集中; PGC/CoFe-TMOs 样品(图 3c)仅在氧化物 颗粒边缘存在少许碳材料, 其中有序层状结构碳的层数 及分布面积都少于 N-PGC/CoFe-TMOs 和 N'-PGC/ CoFe-TMOs 样品, 这与 Raman 光谱图中三样品 D 带、 $G$ 带强度相对大小的结果一致. 通过以上分析可知, CTS 有机前体具有更好的碳化、石墨化特性.

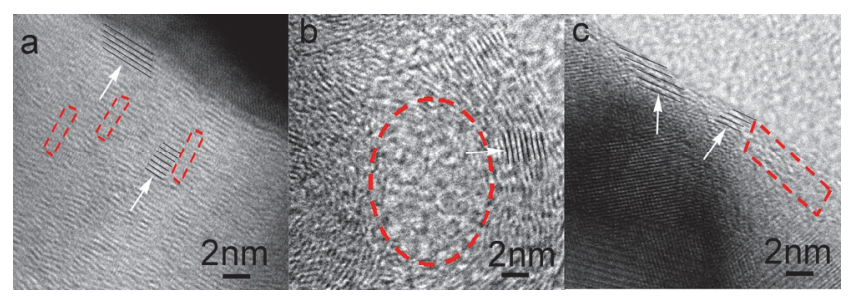

图 3 N-PGC/CoFe-TMOs (a)、N'-PGC/CoFe-TMOs (b)和 PGC/CoFeTMOs (c)样品 HRTEM 图

Figure 3 HRTEM images of N-PGC/CoFe-TMOs (a), N'-PGC/CoFeTMOs (b) and PGC/CoFe-TMOs (c)

\subsection{3 比表面积与孔径分布}

图 4 为部分石墨化碳/钴铁氧化物样品的 $\mathrm{N}_{2}$ 吸附脱附等温线和孔径分布图. 由图 4a 可以看出三种纳米 复合材料的吸附等温线均为第 IV 类吸附等温线. 在低压 下气体吸附量随相对压力缓慢增加, 表明材料中微孔含 量少; 在中、高相对压力区域 $\left(0.45<P / P_{0}<1.0\right)$, 吸附量 随压力显著增加, 气体在孔道中发生凝聚, 并且脱附等 温线与吸附等温线形成滞后圈, 表明复合材料中含有介 孔与大孔孔道 ${ }^{[17]}$. 对于 N-PGC/CoFe-TMOs、PGC/CoFeTMOs 和 N'-PGC/CoFe-TMOs 复合材料, 按照 BET 方法 计算得到的比表面积分别为 $70.4 、 31.1$ 和 $24.0 \mathrm{~m}^{2} \cdot \mathrm{g}^{-1}$, 根据 $\mathrm{BJH}$ 方法分析三种材料的孔体积分别为 $0.33 、 0.27$ 和 $0.18 \mathrm{~cm}^{3} \cdot \mathrm{g}^{-1}$. 图 $4 \mathrm{~b}$ 给出了三种复合材料的孔径分布 图, 按照 BJH方法利用脱附数据分析可得上述三种复合 材料的平均孔径分别为 $13.7 、 24.7$ 和 $14.7 \mathrm{~nm}$. 另外从图 $4 \mathrm{~b}$ 还可以看出 $\mathrm{N}-\mathrm{PGC} / \mathrm{CoFe}-\mathrm{TMOs}$ 纳米复合材料中, 主 要包含了介孔与大孔两种类型的孔径, 集中分布的孔径
尺寸为: 较小的介孔 $7.3 \mathrm{~nm}$ 、中等介孔 $32.2 \mathrm{~nm}$ 和大孔 $120.3 \mathrm{~nm}$. 而另外两种复合物中, 较小的介孔和大孔数 量都较低, 主要孔径尺寸分布在 $30 \mathrm{~nm}$ 附近. 实验数据 表明, N-PGC/CoFe-TMOs 具有较高的比表面积和孔体 积, 有利于电解质与电极材料接触, 并且大孔道可以作 为电解质的存储空间, 介孔有利于缩短电解质与离子的 扩散路径 ${ }^{[12]}$, 便于电子传输.
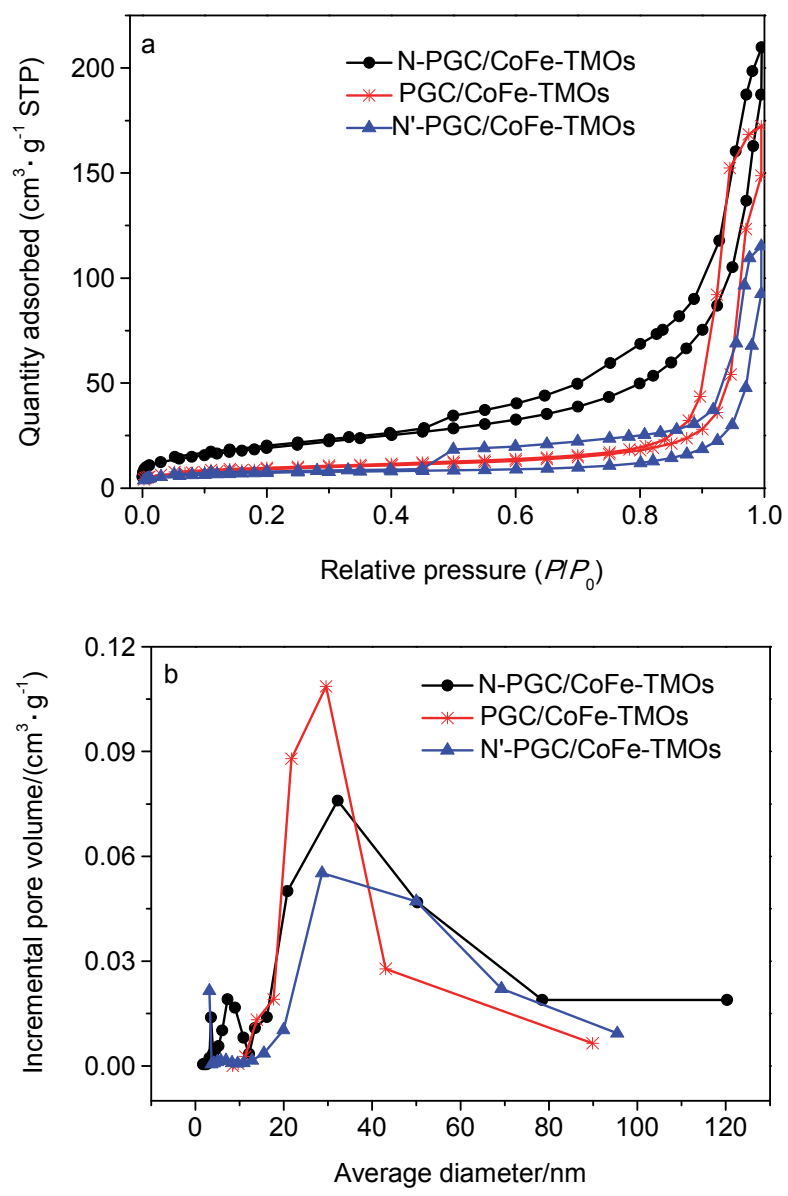

图 4 部分石墨化碳/钴铁氧化物复合材料 $\mathrm{N}_{2}$ 吸附-脱附等温线(a)和孔 径分布图(b)

Figure $4 \quad \mathrm{~N}_{2}$ adsorption-desorption isotherms (a) and pore size distribution curves (b) of PGC/CoFe-TMOs composites

\section{1 .4 扫描电镜(SEM)形貌观测与元素分析}

借助 SEM 分别对有机前体/LDHs 复合物及其衤烧 产物 PGC/TMOs 复合物进行了形貌观测, 如图 5 所示. 从图中可以看出，当采用 CTS 为有机组分时, 制备的 $\mathrm{CTS} / \mathrm{CoFe}-\mathrm{LDHs}$ 复合物具有片层状结构特征 (图 5a), 片层厚度约 $8 \mathrm{~nm}$ (图 $\mathrm{S} 2 \mathrm{~b}$, 支持信息), 而不含有机组分 的纯 CoFe-LDHs 则呈现致密片层与颗粒(粒径约 $28 \mathrm{~nm}$ ) 共存的形貌特征(图 S2a, 支持信息). 分析认为, CTS 中 的羟基、氨基可与 CoFe-LDHs 中的羟基形成氢键从而 得到复合物 ${ }^{[39]}, \mathrm{CTS}$ 与双金属氢氧化物之间的相互作用 可促进 LDHs 颗粒组装与取向, 故 CTS/CoFe-LDHs 片层 结构明显, 且样品厚度变薄. 该结果表明 CTS 对 CoFe- 
LDHs 具有结构导向作用. 当采用阴离子表面活性剂 SDBS 作为有机前体来制备 SDBS/CoFe-LDHs 时, 随着 共沉淀反应进行, 表面活性剂通过静电作用在氢氧化物 表面吸附及层间通道中插层 ${ }^{[40]}$, 生成球形小颗粒(图 $5 b)$, 粒径约 40 50 nm. 当采用 CTS-SDBS 混合物来引 导制备 CTS-SDBS/CoFe-LDHs 材料时, 反应体系中氢 键作用与静电作用同时存在, 产物仍具有明显的片层状 结构特征(图 5c), 表明甲壳胺的调控作用占主导地位. 实验结果表明, 上述三种有机前体可通过不同方式来调 控 LDHs 形貌.

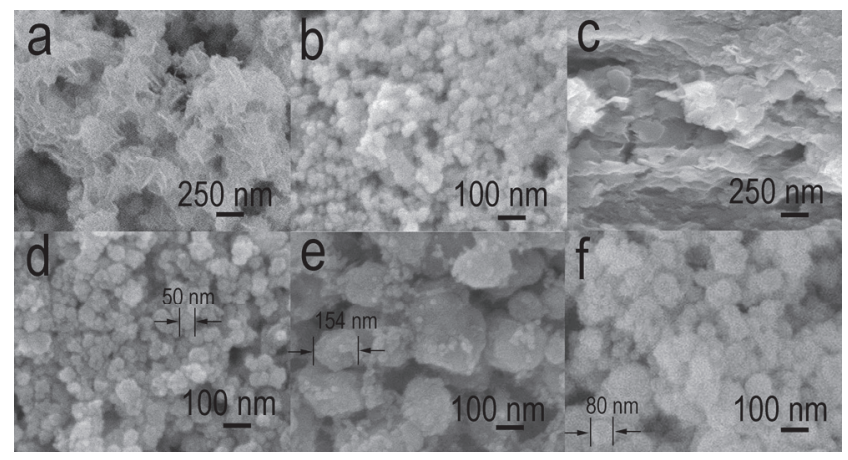

图 $5 \mathrm{CoFe}-\mathrm{LDHs}$ 复合物 $(\mathrm{a}, \mathrm{b}, \mathrm{c})$ 及 $\mathrm{CoFe}-\mathrm{TMOs}$ 复合物 $(\mathrm{d}, \mathrm{e}, \mathrm{f}) \mathrm{SEM}$ 图 Figure 5 SEM images of CoFe-LDHs composites (a, b, c) and corresponding pyrolyzed CoFe-TMOs composites $(\mathrm{d}, \mathrm{e}, \mathrm{f})$

(a) CTS/CoFe-LDHs, (b) SDBS/CoFe-LDHs, (c) CTS-SDBS/CoFe-LDHs, (d) N-PGC/CoFe-TMOs, (e) PGC/CoFe-TMOs, (f) N'-PGC/CoFe-TMOs

有机前体 $/ \mathrm{CoFe}-\mathrm{LDHs}$ 复合物于氩气氛、空气氛中 煅烧后生成 PGC/CoFe-TMOs 黑色固体粉末, SEM 照片 显示产物形貌为球形颗粒. 从图 $5 \mathrm{~d} \sim 5 \mathrm{f}$ 可以看出, N-PGC/CoFe-TMOs、PGC/CoFe-TMOs 和 N'-PGC/CoFe$\mathrm{TMOs}$ 复合物的典型尺寸分别为 $50 、 154$ 和 $80 \mathrm{~nm}$. 另 外, SDBS 有机前体对应的 PGC/CoFe-TMOs 复合物中产 物粒径分布不够均匀, 除大颗粒外, 体系中还有粒径在 $20 \mathrm{~nm}$ 左右的小颗粒, 故 PGC/CoFe-TMOs 比表面积 (31.1 $\mathrm{m}^{2} \cdot \mathrm{g}^{-1}$ )略大于 $\mathrm{N}^{\prime}-\mathrm{PGC} / \mathrm{CoFe}-\mathrm{TMOs}$ 样品 (24.0 $\left.\mathrm{m}^{2} \cdot \mathrm{g}^{-1}\right)$, 它们均小于 N-PGC/CoFe-TMOs 复合物的比表 面积 $\left(70.4 \mathrm{~m}^{2} \cdot \mathrm{g}^{-1}\right)$. 分析认为, SDBS 疏水链包括含十二 个碳原子的脂肪烃链, 柔性较强, 热解产物碳含量低 (4.63 wt\%)导致产物易聚集, 生成的颗粒尺寸较大(图 $5 \mathrm{e})$; 甲壳胺为氨基葡萄糖聚合物, 葡萄糖六元环相对于 脂肪烃链具有一定的刚性, CTS/CoFe-LDHs 煅烧后生成 的 N-PGC/CoFe-TMOs 复合物保持了较好的分散性, 碳 含量可达 $14.61 \mathrm{wt} \%$, 颗粒平均粒径较小(图 5d). 可见, 有机前体结构对于 PGC/CoFe-TMOs 颗粒尺寸有重要影 响. 另外, 本文还对比观测了有机前体/CoFe LDHs 在 氩气氛中炦烧产物的形貌, 如图 S3 (支持信息)所示. 从 图中可以看出, LDHs 复合物经过氩气氛煅烧过程就已 基本形成最终产物形貌特征, 样品再经过空气氛低温 $\left(250{ }^{\circ} \mathrm{C}\right)$ 㷽烧, 颗粒尺寸无明显变化, 空气氛煅烧的主 要作用体现在过渡金属合金或单质转变为氧化物. 能量弥散谱(energy dispersive spectrum, EDS)实验 结果显示, N-PGC/CoFe-TMOs 和 N'-PGC/CoFe-TMOs 样品所含主要元素为 $\mathrm{C} 、 \mathrm{~N} 、 \mathrm{O} 、 \mathrm{Co}$ 和 $\mathrm{Fe}, \mathrm{PGC} / \mathrm{CoFe}-$ TMOs 主要含有 $\mathrm{C} 、 \mathrm{O} 、 \mathrm{Co}$ 和 $\mathrm{Fe}$ 元素, 未检测出 $\mathrm{N}$ 元 素. 其中, N-PGC/CoFe-TMOs 样品对应 C、N、O、Co 和 $\mathrm{Fe}$ 元素质量分数 $(\mathrm{wt} \%)$ 分别为 $14.61 \% 、 0.98 \%$ 、 $27.08 \% 、 19.45 \%$ 和 $37.87 \%$ (样品 EDS 谱图如图 S4 所示, 支持信息), 而根据元素分析测试得到样品中 $\mathrm{C} 、 \mathrm{~N}$ 元素 质量分数分别为 $13.87 \%$ 和 $0.89 \%$, 两种方法测试结果接 近，据此计算样品中 N/C 原子比例约为 $1 / 18$. 从图 6 扫 描电镜照片与元素分布图可以看出, 上述各种元素在样 品中分布均匀. N'-PGC/CoFe-TMOs 中 C、N 元素质量分 数分别为 $5.60 \%$ 和 $1.39 \%$, 样品中碳元素含量明显降低 (小于 N-PGC/CoFe-TMOs 样品碳含量的一半), 表明生 物聚合物 CTS 比小分子表面活性剂 SDBS 具有更好的 碳化性能. 该实验结果显示, 含氮有机前体 CTS 经过惰 性气氛耘烧成功实现碳材料原位氮掺杂, 在此基础上与 钴铁氧化物形成纳米复合材料.

\section{2 电化学性能表征}

为考察敾烧产物作为超级电容器电极材料的电化 学性能, 实验采用三电极体系, 在 $6 \mathrm{~mol} \bullet \mathrm{L}^{-1} \mathrm{KOH}$ 溶液 中对上述纳米复合物进行了循环伏安扫描和恒电流充 放电测试, 并分析比电容大小, 考察电极材料倍率特性 和循环稳定性.

\subsection{1 循环伏安曲线}

以 CTS 为有机前体, 分别制备了含不同金属元素 的氮掺杂部分石墨化碳/氧化物复合材料, 包括 N-PGC/ CoFe-TMOs、N-PGC/NiFe-TMOs、N-PGC/CoAl-TMOs 和 N-PGC/NiAl-TMOs. 它们的循环伏安 $(\mathrm{CV})$ 曲线如图 7 所示. 从图中可以看出, 扫描速率为 $5 \mathrm{mV} \cdot \mathrm{s}^{-1}$ 时, 在 $0 \sim 0.45 \mathrm{~V}$ vs. SCE 区间范围内, 复合物在扫描过程中均 具有典型的氧化峰 $(0.30 \sim 0.40 \mathrm{~V})$ 和还原峰 $(0.20 \sim 0.35$ $\mathrm{V})$, 与纯碳材料矩形 $\mathrm{CV}$ 曲线显著不同, 表明复合物具 有典型的噟电容特性. 含 $\mathrm{Co}_{3} \mathrm{O}_{4} 、 \mathrm{Fe}_{3} \mathrm{O}_{4} 、 \mathrm{NiO}$ 等氧化物 及其复合物的电极材料，其氧化、还原过程对应的反应 方程式可表示为 ${ }^{[3,4,26,41]}$ :

$$
\begin{aligned}
& \mathrm{Co}_{3} \mathrm{O}_{4}+\mathrm{OH}^{-}+\mathrm{H}_{2} \mathrm{O} \leftrightarrow 3 \mathrm{CoOOH}+\mathrm{e}^{-} \\
& \mathrm{CoOOH}+\mathrm{OH}^{-} \leftrightarrow \mathrm{CoO}_{2}+\mathrm{H}_{2} \mathrm{O}+\mathrm{e}^{-} \\
& 2 \mathrm{Fe}_{3} \mathrm{O}_{4}+2 \mathrm{OH}^{-} \leftrightarrow 3 \mathrm{Fe}_{2} \mathrm{O}_{3}+\mathrm{H}_{2} \mathrm{O}+2 \mathrm{e}^{-} \\
& \mathrm{NiO}+\mathrm{OH}^{-} \leftrightarrow \mathrm{NiOOH}+\mathrm{e}^{-}
\end{aligned}
$$

2.2.2 恒电流充放电 (galvanostatic charge discharge, GCD)

在 $2 \mathrm{~A} \cdot \mathrm{g}^{-1}$ 电流密度下，含不同金属元素的 $\mathrm{N}-\mathrm{PGC} / \mathrm{TMOs}$ 复合物 GCD 曲线如图 8a 所示. 该充放电 曲线形状不是对称三角形, 在充放电过程都存在工作电 极电势上升、下降的平缓区或平台区，其电势范围与 $\mathrm{CV}$ 曲线中氧化峰、还原峰电极电势范围相对应. GCD 曲线 


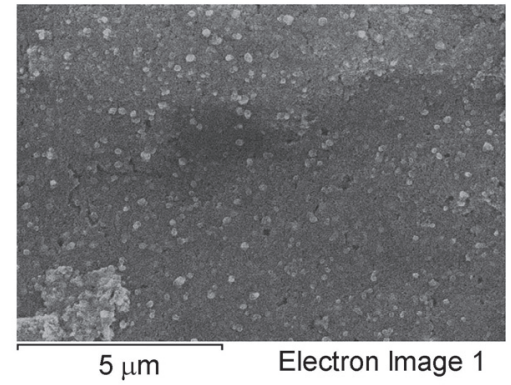

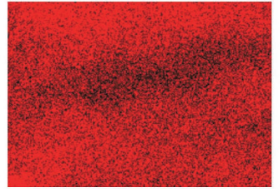

$\mathrm{C} K \alpha 1 \_2$

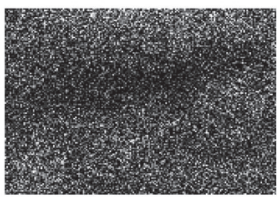

$\mathrm{OK} \alpha \mathrm{I}$

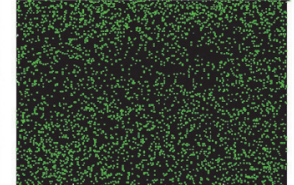

N Ko1_2

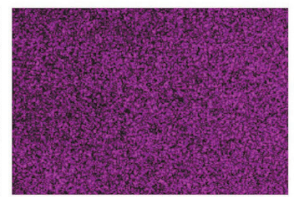

Co Kal

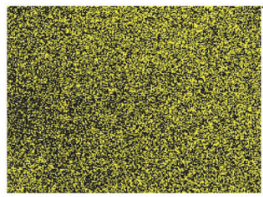

$\mathrm{Fe} \mathrm{K} \alpha 1$

图 $6 \mathrm{~N}-\mathrm{PGC} / \mathrm{CoFe}-\mathrm{TMO}$ 复合物 SEM 照片与元素分布图

Figure 6 SEM image of N-PGC/CoFe-TMOs composite and element mapping

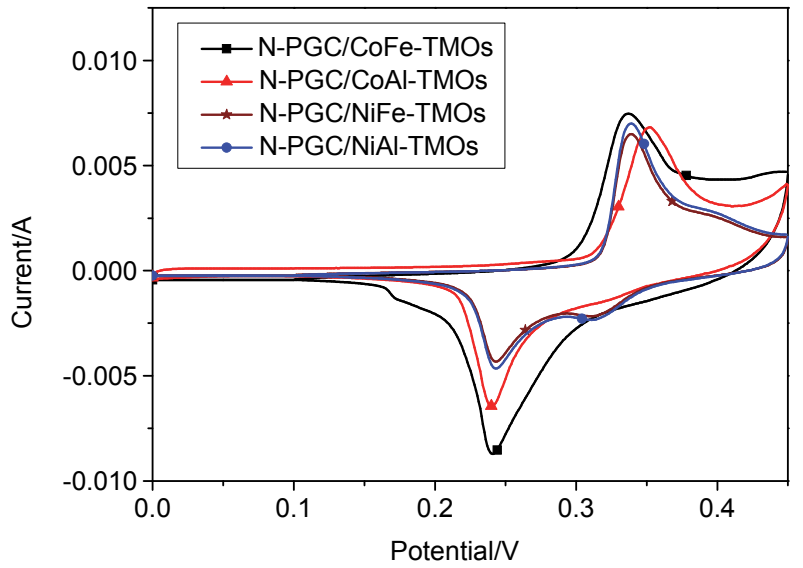

图 7 含不同金属元素的 N-PGC/TMOs 循环伏安曲线

Figure $7 \mathrm{CV}$ curves of N-PGC/TMOs containing different metal elements

中, 工作电极电势与时间成线性关系时, 对应于电解质 离子在电解质溶液/电极材料界面上物理吸附与脱附过 程，电极电势变化平缓区则对应于电极表面的氧化还原
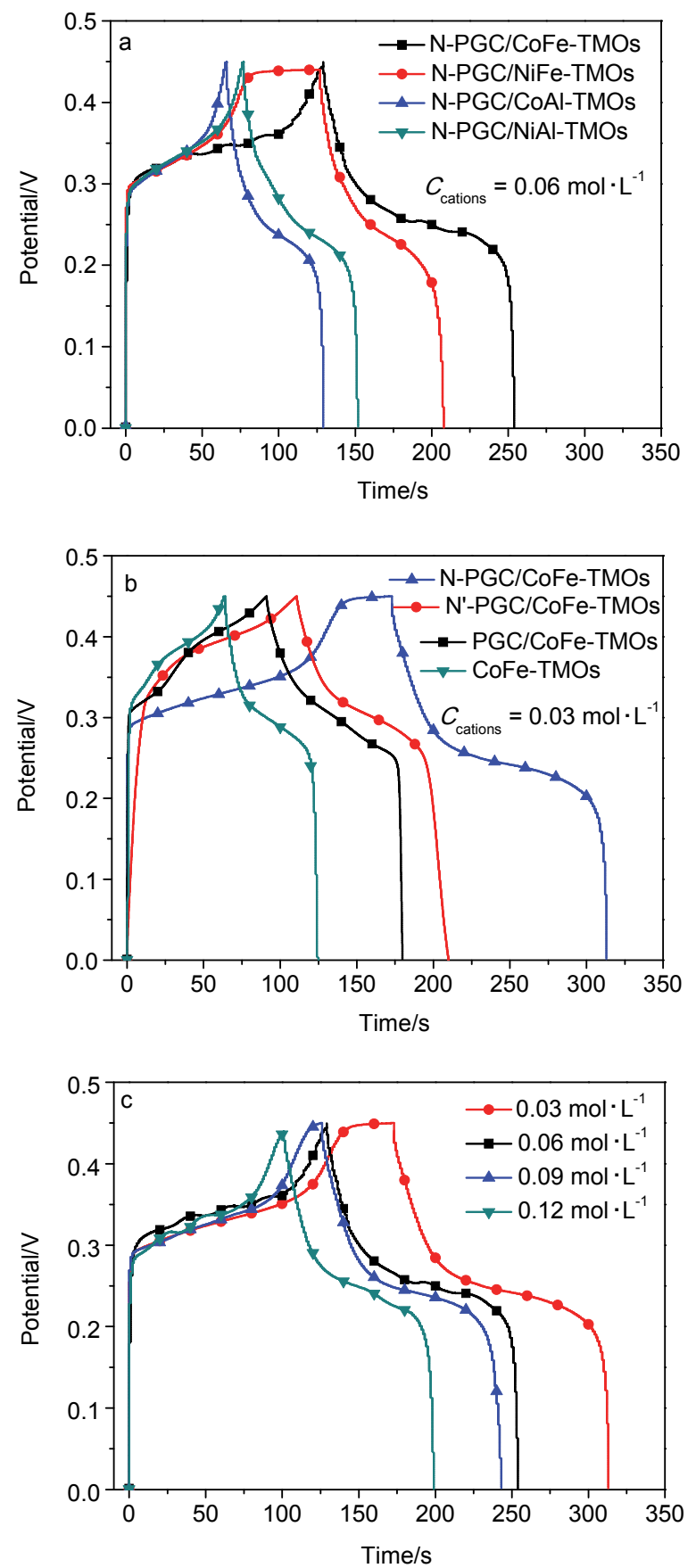

图 $82 \mathrm{~A} \cdot \mathrm{g}^{-1}$ 电流密度下过渡金属氧化物及其复合物 $\mathrm{GCD}$ 曲线 (a)含不同金属元素的复合物 $\left(C_{\mathrm{cations}}=0.06 \mathrm{~mol} \cdot \mathrm{L}^{-1}\right),(\mathrm{b})$ 不同有机前体衍生 碳/钴铁氧化物复合物及纯钴铁氧化物 $\left(C_{\mathrm{cations}}=0.03 \mathrm{~mol} \cdot \mathrm{L}^{-1}\right),(\mathrm{c})$ 不同

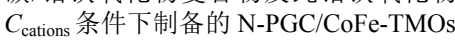

Figure 8 GCD curves of transition metal oxides and their composites at the current density of $2 \mathrm{~A} \cdot \mathrm{g}^{-1}$

(a) composites containing different metal elements $\left(C_{\text {cations }}=0.06 \mathrm{~mol} \cdot \mathrm{L}^{-1}\right)$, (b) various organic precursor derived carbon/CoFe-TMOs composites and pure CoFe-TMOs $\left(C_{\text {cations }}=0.03 \mathrm{~mol} \cdot \mathrm{L}^{-1}\right)$, (c) N-PGC/CoFe-TMOs prepared at different $C_{\text {cations }}$ circumstances

反应过程 ${ }^{[23,25]}$. 因此, 根据充放电曲线可以判断 $\mathrm{N}-\mathrm{PGC} / \mathrm{TMOs}$ 同时具有赝电容特性与双电层电容器特 性，并且噟电容是纳米复合电极材料储能的重要方式. 另外，根据 N-PGC/CoFe-TMOs、N-PGC/NiFe-TMOs、 
N-PGC/CoAl-TMOs 和 N-PGC/NiAl-TMOs 样品放电曲 线实验数据, 计算得到它们的比电容分别为 568.9、 446.5、291.1 和 $328.9 \mathrm{~F} \bullet \mathrm{g}^{-1}$. 起始溶液发生共沉淀反应 时, 阳离子浓度 $C_{\text {cations }}$ 为 $0.06 \mathrm{~mol} \cdot \mathrm{L}^{-1}$. 在相同实验条件 下, 钴铁氧化物表现出更高的氧化还原活性. 图 $8 \mathrm{~b}$ 给出 了复合物 N-PGC/CoFe-TMOs、N'-PGC/CoFe-TMOs、 PGC/CoFe-TMOs 及纯氧化物 CoFe-TMOs 的 GCD 曲线. 发生溶液共沉淀反应时, $C_{\text {cations }}$ 为 $0.03 \mathrm{~mol} \cdot \mathrm{L}^{-1}$. 实验条 件下，上述四样品的比电容分别是 671.1、440.5、410.0 和 $283.3 \mathrm{~F} \cdot \mathrm{g}^{-1}$. 一方面, 与纯氧化物相比, 复合材料均 具有较高的比电容. 这可能是有机前体能够对类水滑石 材料结构与尺度进行调控, 二者可实现分子级别上的分 散. 煆烧过程中碳材料与氧化物互为分散剂, 颗粒聚集 程度降低, 电极材料死体积比例减少, 活性物质可以更 充分地参与电化学反应. 同时, 过渡金属对碳材料还具

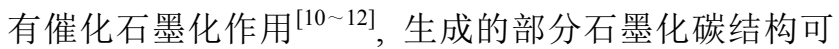
以提高复合物的导电性能. 另一方面, 就复合物而言, 碳材料中掺杂氮元素会显著提高复合材料比电

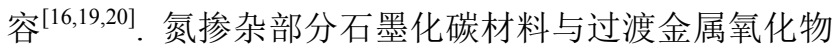
复合具有更加明显的协同效应.

在优选无机、有机前体种类的基础上，固定甲壳胺 浓度与用量, 通过改变共沉淀反应中阳离子总浓度 $C_{\text {cations }}\left(0.03 \sim 0.12 \mathrm{~mol} \cdot \mathrm{L}^{-1}\right)$ 来探索 $\mathrm{N}-\mathrm{PGC} / \mathrm{CoFe}-\mathrm{TMOs}$ 产物电化学性能变化规律. 各产物在 $2 \mathrm{~A} \cdot \mathrm{g}^{-1}$ 电流密度 下的 GCD 曲线如图 8c 所示. 实验结果表明, 钴、铁阳 离子总浓度为 $0.03 、 0.06 、 0.09$ 和 $0.12 \mathrm{~mol} \cdot \mathrm{L}^{-1}$ 时, 对应 产物比电容分别为 $671.1 、 568.9 、 542.2$ 和 $457.3 \mathrm{~F}^{\circ} \mathrm{g}^{-1}$. 分 析认为, 实验条件下随着共沉淀反应体系中金属盐浓度 减小, 氢氧化物前体及最终的氧化物纳米复合材料可能 具有更高的比表面积, 颗粒的聚集程度相对较小, 电活 性组分能够更充分地与电解液接触并参与电化学反应, 因此表现出较好的电化学性能.

\subsection{3 倍率特性与循环稳定性}

倍率特性与循环稳定性也是 $\mathrm{SC}$ 电极材料的重要性 能. 针对在 $C_{\text {cations }}$ 为 $0.03 \mathrm{~mol} \cdot \mathrm{L}^{-1}$ 条件下制备的 N-PGC/ CoFe-TMOs 样品, 分别在 $1 、 2 、 5 、 8$ 和 $10 \mathrm{~A} \cdot \mathrm{g}^{-1}$ 电流 密度下进行充放电测试，其比电容分别为 737.8、671.1、 640.0、600.0 和 $573.3 \mathrm{~F} \cdot \mathrm{g}^{-1}$, 如图 9 所示. 当电流密度从 $1 \mathrm{~A} \cdot \mathrm{g}^{-1}$ 增加至 $2 \mathrm{~A} \cdot \mathrm{g}^{-1}$ 时, 纳米复合物比电容下降了 $66.8 \mathrm{~F} \cdot \mathrm{g}^{-1}$, 说明随电流密度增加电极材料电化学反应 不可逆程度增加. 继续增加电流密度时, 比电容-电流 密度曲线斜率变缓. 分析认为, 复合物中氮掺杂部分石 墨化碳材料可促进电子传输, 该结构可减缓高电流密度 下电极材料比容量衰减. 电流密度扩大十倍至 $10 \mathrm{~A} \cdot \mathrm{g}^{-1}$ 时, 复合物比电容保留率为 $77.7 \%$. 另外对 $\mathrm{N}-\mathrm{PGC} / \mathrm{CoFe}-\mathrm{TMOs}$ 样品在 $10 \mathrm{~A} \cdot \mathrm{g}^{-1}$ 电流密度下进行了 循环稳定性测试, 结果如图 10 所示. 扫描次数低于 300 次时, 随着充放电过程进行, 电解液在电极材料中充分
扩散并与之接触, 样品比电容略有增加. 电极材料经过 5000 次循环充放电, 其比电容为 $380.7 \mathrm{~F}^{\circ} \mathrm{g}^{-1}$, 保留率为 $66.4 \%$. 以上实验数据表明, N-PGC/CoFe-TMOs 纳米复 合物作为 $\mathrm{SC}$ 电极材料具有良好的倍率特性与循环稳定 性.

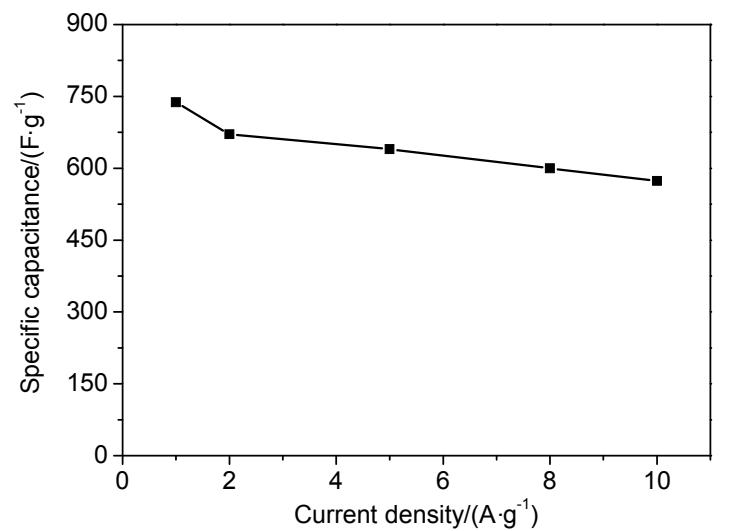

图 $9 \mathrm{~N}-\mathrm{PGC} / \mathrm{CoFe}-\mathrm{TMOs}$ 比电容-电流密度曲线 $\left(C_{\text {cations }}=0.03 \mathrm{~mol} \cdot \mathrm{L}^{-1}\right)$ Figure 9 Specific capacitance-current density curve of N-PGC/CoFeTMOs sample $\left(C_{\text {cations }}=0.03 \mathrm{~mol} \cdot \mathrm{L}^{-1}\right)$

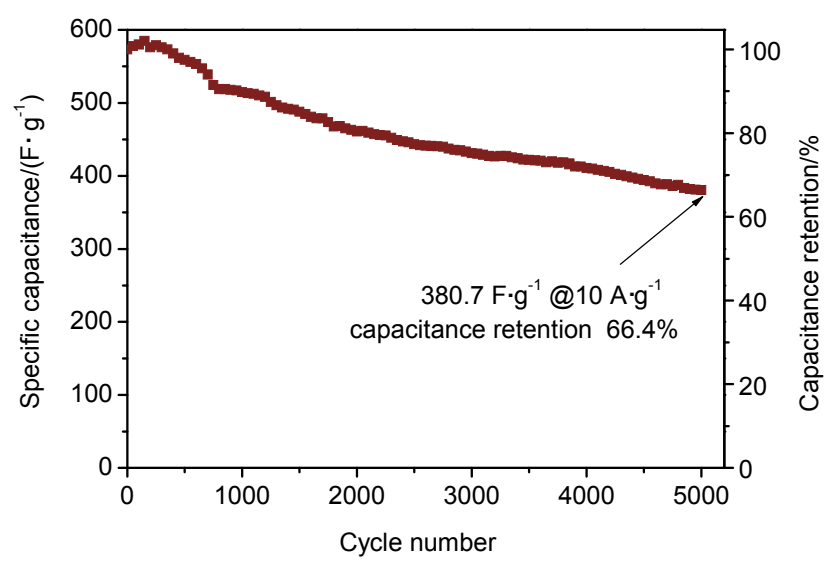

图 $1010 \mathrm{~A} \cdot \mathrm{g}^{-1}$ 电流密度下 $\mathrm{N}-\mathrm{PGC} / \mathrm{CoFe}-\mathrm{TMOs}$ 循环性能曲线 Figure 10 Cycling performance of N-PGC/CoFe-TMOs sample at the constant current density of $10 \mathrm{~A} \cdot \mathrm{g}^{-1}$

\section{3 结论}

本文提出一种构建氮掺杂部分石墨化碳/过渡金属 氧化物纳米复合材料的简便、高效和通用方法. 即采用 含氮元素的生物聚合物甲壳胺为结构导向剂和有机前 体，以过渡金属盐为反应物，通过共沉淀反应制备有机 物/LDHs 复合物, 样品经过惰性气氛与空气氛炦烧, 利 用过渡金属催化石墨化作用成功制备了氮掺杂部分石 墨化碳/过渡金属氧化物纳米复合物. 碳材料可有效防 止氧化物聚集，产物具有较高的比表面积. 复合物中大 孔可以作为电解质的缓冲空间，介孔可以缩短电解质离 子与电子的扩散路径，电活性组分利用率更高. 另外, 氮掺杂石墨化碳还可以促进提高复合材料的导电性与 电化学活性. 以氮掺杂部分石墨化碳/过渡金属氧化物 
纳米复合物作 $\mathrm{SC}$ 电极材料, 兼有双电层电容特性与赝 电容特性, 该复合物具有较高的比电容和良好的倍率特 性与循环稳定性, 在超级电容器领域应用前景广阔.

\section{4 实验部分}

\section{$4.1 \mathrm{LDHs}$ 复合物制备}

CTS/LDHs 复合材料制备: 配制 $250 \mathrm{~mL} 0.06 \mathrm{~mol} \bullet$ $\mathrm{L}^{-1}$ 的醋酸溶液, 向其中加入 $2.240 \mathrm{~g} \mathrm{CTS}$, 搅拌至完全 溶解, 然后根据材料种类和浓度要求加入一定量金属硝 酸盐, 最后滴加 $50 \mathrm{~mL}$ 氢氧化钠溶液通过共沉淀反应制 备 CTS/LDHs 目标产物. 实验过程中, 控制溶液中二价、 三价阳离子物质的量之比 $n_{\mathrm{M} 2}+n_{\mathrm{M} 3}+$ 为 $2 / 1$, 沉淀剂中 $\mathrm{OH}^{-}$的物质的量为理论值的 1.2 倍, 调节反应液 $\mathrm{pH}$ 值在 9 附近. 上述体系在 $70{ }^{\circ} \mathrm{C}$ 、搅拌条件下反应 $15 \mathrm{~h}$. 反应 结束后分别用水和乙醇对样品进行抽滤、洗涤三次, 在 $70{ }^{\circ} \mathrm{C}$ 下真空干燥 $6 \mathrm{~h}$, 得到复合材料.

为考察不同种类阳离子对产物电化学性能的影响, 溶液进行共沉淀反应时, 在阳离子总浓度 $\left(C_{\text {cations }}\right)$ 为 0.06 $\mathrm{mol} \cdot \mathrm{L}^{-1}$ 条件下分别制备了 $\mathrm{CTS} / \mathrm{CoFe}-\mathrm{LDHs}$ 、 CTS/NiFe-LDHs、CTS/CoAl-LDHs 和 CTS/NiAl-LDHs 复合材料; 为分析电极材料随起始反应物浓度的变化规 律, 另在 $C_{\text {cations }}$ 为 $0.03 、 0.09$ 和 $0.12 \mathrm{~mol} \cdot \mathrm{L}^{-1}$ 条件下分 别制备了 $\mathrm{CTS} / \mathrm{CoFe}-\mathrm{LDHs}$.

为对比有机前体对产物结构与性能的影响, 在 $C_{\text {cations }}$ 为 $0.03 \mathrm{~mol} \cdot \mathrm{L}^{-1}$ 条件下, 还分别选用阴离子表面 活性剂十二烷基苯磺酸钠(SDBS)、CTS-SDBS 混合物为 结构导向剂制备了 SDBS/CoFe-LDHs 和 CTS-SDBS/ $\mathrm{CoFe}-\mathrm{LDHs}$ 复合材料. 以 SDBS 为结构导向剂时, 控制 反应物配比 $n_{\mathrm{Fe} 3} / n_{\mathrm{SDBS}}$ 为 $2 / 1$, 选用混合物作结构导向剂 时, CTS 与 SDBS 浓度均为相应单一结构导向剂浓度的 一半. 作为对比, 在相同操作条件下还制备了不含有机 组分的 CoFe-LDHs 纯氢氧化物.

\section{2 过渡金属氧化物(TMOs)复合物制备}

将 LDHs 样品置于瓷舟中放入石英管式炉，调整氩 气流量为 $150 \mathrm{~cm}^{3} \cdot \mathrm{min}^{-1}$, 通气 $0.5 \mathrm{~h}$ 以排除管式炉中的 空气. 然后将氩气流量调为 $100 \mathrm{~cm}^{3} \cdot \mathrm{min}^{-1}$, 以 $5{ }^{\circ} \mathrm{C} \cdot$ $\mathrm{min}^{-1}$ 的升温速率升温至 $200{ }^{\circ} \mathrm{C}$ 并恒温 $1 \mathrm{~h}$, 再以相同的 升温速率升温至 $600{ }^{\circ} \mathrm{C}$, 恒温 $2 \mathrm{~h}$ 后关闭热源. 待管式 炉冷却到室温, 关闭氩气源. 为使金属元素充分氧化, 在空气气氛中, 以 $5{ }^{\circ} \mathrm{C} \cdot \mathrm{min}^{-1}$ 的升温速率将样品再次加 热至 $250{ }^{\circ} \mathrm{C}$, 恒温 $12 \mathrm{~h}$ 得目标产物. CTS/LDHs、CTSSDBS/LDHs、SDBS/LDHs 和 LDHs 耘烧产物分别记为 N-PGC/TMOs、N'-PGC/TMOs、PGC/TMOs 和 TMOs.

\section{3 结构与形貌表征}

利用日本理学公司 D/MAX-2500/PC 型 X 射线衍射

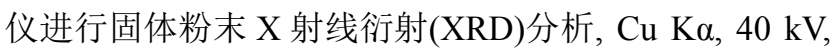
$150 \mathrm{~mA}, 2 \theta$ 范围 $3^{\circ} \sim 80^{\circ}$, 扫描速度 $8\left(^{\circ}\right) \cdot \mathrm{min}^{-1}$. 采用英
国雷尼绍公司 Renishaw In Via 激光拉曼光谱仪记录样 品拉曼光谱，激发波长为 $632.8 \mathrm{~nm}$ ，扫描范围 1000 $2000 \mathrm{~cm}^{-1}$. 使用美国麦克仪器公司 ASAP2020 物理吸 附仪在 $77 \mathrm{~K}$ 下进行 $\mathrm{N}_{2}$ 吸附-脱附等温曲线研究, 分别根 据 BET 方法、BJH 方法计算比表面积与平均孔径. 通过 日本电子公司 JSM-6700F 型扫描电子显微镜(SEM)、 JEM-2100Plus 透射电子显微镜(TEM) 观测样品形貌, 借助 SEM 上配置的英国牛津公司能谱仪 X-MAX 进行 样品元素分析并观察其分布特征，通过 Vario EL III 元 素分析仪测定了样品中 $\mathrm{C} 、 \mathrm{~N}$ 元素含量.

\section{4 纳米复合物电极制作与电化学性能测试}

采用上述碳/过渡金属氧化物纳米复合物涂制工作 电极, 通过 CHI660D 电化学工作站于 $25{ }^{\circ} \mathrm{C} 6 \mathrm{~mol} \cdot \mathrm{L}^{-1}$ $\mathrm{KOH}$ 溶液中测试了产物的电化学性能. 其中工作电极 的制备是按照活性物：导电剂乙炔黑：粘结剂(聚四氟 乙烯乳液, 质量分数 5\%)质量比为 $80: 10: 10$ 取样, 用 少量 $N$-甲基吡咯烷酮(NMP)调浆, 并涂覆于泡沫镍集流 体 $(1 \mathrm{~cm} \times 1 \mathrm{~cm})$ 上. 于烘箱中 $70{ }^{\circ} \mathrm{C}$ 干燥 $2 \mathrm{~h}$, 然后 $100{ }^{\circ} \mathrm{C}$ 真空干燥 $12 \mathrm{~h}$, 以完全去除 NMP. 根据泡沫镍涂 覆浆液前后质量变化, 计算活性物质承载量. 本实验中 控制活性物质承载量在 $0.6 \sim 0.9 \mathrm{mg} \cdot \mathrm{cm}^{-2}$ 范围. 以制备 的工作电极、饱和甘录电极 $(\mathrm{SCE}$, 参比电极)、铂片 $(1 \mathrm{~cm}$ $\times 2 \mathrm{~cm}$, 对电极)构建三电极体系进行电化学测试, 包括 循环伏安 $(C V)$ 测试和恒电流充放电 $(\mathrm{GCD})$ 测试. $\mathrm{CV}$ 测 试电压范围为 $0 \sim 0.45 \mathrm{~V}$ vs. SCE, 扫描速率为 $5 \mathrm{mV} \cdot \mathrm{s}^{-1}$. 选用不同电流密度, 在 $0 \sim 0.45 \mathrm{~V} v \mathrm{Vs}$. SCE 的电压范围内 进行恒电流充放电分析，计算产物比电容，并考察其循 环稳定性与倍率特性. 比电容计算公式为:

$$
C=\frac{I \Delta t}{m \Delta V}
$$

其中, $I$ 代表测试过程的充放电电流(A), $\Delta t$ 为放电时间 $(\mathrm{s}), m$ 为工作电极上活性材料的质量 $(\mathrm{g}), \Delta V$ 为总的电 压降(V).

\section{References}

[1] Miller, J. R.; Simon, P. Science 2008, 321, 651.

[2] Simon, P.; Gogotsi, Y. Nature Mater. 2008, 7, 845

[3] Wang, G.; Zhang, L.; Zhang, J. Chem. Soc. Rev. 2012, 41, 797.

[4] Zhang, Y.; Feng, H.; Wu, X.; Wang, L.; Zhang, A.; Xia, T.; Dong, H.; Li, X.; Zhang, L. Int. J. Hydrogen Energy 2009, 34, 4889.

[5] Li, X.-Q.; Chang, L.; Zhao, S.-L.; Hao, C.-L.; Lu, C.-G.; Zhu, Y.-H.; Tang, Z.-Y. Acta Phys.-Chim. Sinica 2017, 33, 130. (李雪芹, 常琳, 赵慎龙, 郝昌龙, 陆晨光, 朱以华, 唐智勇, 物理化学学报, 2017, 33, 130.)

[6] Liu, Z.; Chen, W.; Fan, X.; Yu, J.; Zhao, Y. Chin. J. Chem. 2016, 34 839.

[7] Wu, J.; Zhou, A.; Huang, Z.; Li, L.; Bai, H. Chin. J. Chem. 2016, 34, 67.

[8] Yang, J.; Yu, C.; Fan, X.; Liang, S.; Li, S.; Huang, H.; Ling, Z.; Hao, C.; Qiu, J. Energy Environ. Sci. 2016, 9, 1299.

[9] Staaf, L. G. H.; Lundgren, P.; Enoksson, P. Nano Energy 2014, 9, 128.

[10] Mun, Y.; Jo, C.; Hyeon, T.; Lee, J.; Ha, K.-S.; Jun, K.-W.; Lee, S.-H.; Hong, S.-W.; Lee, H. I.; Yoon, S.; Lee, J. Carbon 2013, 64, 
391.

[11] Gao, W.; Wan, Y.; Dou, Y.; Zhao, D. Adv. Energy Mater. 2011, 1, 115.

[12] Wang, D.-W.; Li, F.; Liu, M.; Lu, G. Q.; Cheng, H.-M. Angew. Chem. Int. Ed. 2008, 47, 373.

[13] Lin, T.; Chen, I.-W.; Liu, F.; Yang, C.; Bi, H.; Xu, F.; Huang, F. Science 2015, 350, 1508.

[14] Yang, M.; Zhong, Y.; Bao, J.; Zhou, X.; Wei, J.; Zhou, Z. J. Mater. Chem. A 2015, 3, 11387.

[15] Su, S.; Lai, Q.; Liang, Y. Acta Chim. Sinica 2015, 73, 735. (苏善金, 来庆学, 梁彦瑜, 化学学报, 2015, 73, 735.)

[16] Li, Z.-H.; Li, S.-J.; Zhou, J.; Zhu, T.-T.; Shen, H.-L.; Zhuo, S.-P. Acta Phys.-Chim. Sinica 2015, 31, 676. (李朝辉, 李仕蛟, 周晋, 朱 婷婷, 沈红龙, 禚淑萍, 物理化学学报, 2015, 31, 676.)

[17] Titirici, M.-M.; White, R. J.; Brun, N.; Budarin, V. L.; Su, D. S.; Monte, F. D.; Clark, J. H.; MacLachlan, M. J. Chem. Soc. Rev. 2015, 44, 250.

[18] Primo, A.; Atienzar, P.; Sanchez, E.; Delgado, J. M.; García, H. Chem. Commun. 2012, 48, 9254.

[19] Hao, P.; Zhao, Z.; Leng, Y.; Tian, J.; Sang, Y.; Boughton, R. I.; Wong, C. P.; Liu, H.; Yang, B. Nano Energy 2015, 15, 9.

[20] Tong, X.; Zhuo, H.; Wang, S.; Zhong, L.; Hu, Y.; Peng, X.; Zhou, W.; Sun, R. RSC Adv. 2016, 6, 34261 .

[21] Rybarczyk, M. K.; Lieder, M.; Jablonska, M. RSC Adv. 2015, 5, 44969.

[22] Shao, M.; Zhang, R.; Li, Z.; Wei, M.; Evans, D. G.; Duan, X. Chem. Commun. 2015, 51, 15880.

[23] Yang, Q.; Lu, Z.; Liu, J.; Lei, X.; Chang, Z.; Luo, L.; Sun, X. Prog. Nat. Sci.: Mater. Int. 2013, 23, 351.

[24] Zhao, M.-Q.; Zhang, Q.; Huang, J.-Q.; Wei, F. Adv. Funct. Mater. 2012, 22, 20.

[25] Yan, L.; Kong, H.; Li, Z. Acta Chim. Sinica 2013, 71, 822. (严琳, 孔惠, 李在均, 化学学报, 2013, 71, 822.)

[26] Jeong, G. H.; Baek, S.; Lee, S.; Kim, S.-W. Chem. Asian J. 2016, 11,
949.

[27] Jia, W.; Xu, M.; Lei, C.; Bao, S.; Jia, D. Acta Chim. Sinica 2011, 69 , 1773. (贾巍, 徐茂文, 雷超, 包淑娟, 贾殿赠, 化学学报, 2011 , 69, 1773.)

[28] Chen, Y.; Zhang, Z.-L.; Sui, Z.-J.; Liu, Z.-T.; Zhou, J.-H.; Zhou, X.-G. Acta Phys.-Chim. Sinica 2015, 31, 1105. (陈阳, 张梓澜, 隋 志军, 刘芝婷, 周静红, 周兴贵, 物理化学学报, 2015, 31, 1105.)

[29] Yang, J.; Yu, C.; Fan, X.; Zhao, C.; Qiu, J. Adv. Funct. Mater. 2015, 25, 2109.

[30] Yang, J.; Yu, C.; Fan, X.; Qiu, J. Adv. Energy Mater. 2014, 1400761.

[31] Yu, C.; Yang, J.; Zhao, C.; Fan, X.; Wang, G.; Qiu, J. Nanoscale 2014, 6, 3097.

[32] Yang, J.; Yu, C.; Fan, X.; Ling, Z.; Qiu, J.; Gogotsi, Y. J. Mater. Chem. A 2013, 1, 1963.

[33] Xu, J.; He, F.; Gai, S.; Zhang, S.; Li, L.; Yang, P. Nanoscale 2014, 6, 10887.

[34] Hao, P.; Zhao, Z.; Li, L.; Tuan, C.-C.; Li, H.; Sang, Y.; Jiang, H.; Wong, C. P.; Liu, H. Nanoscale 2015, 7, 14401.

[35] Zhang, H.-J.; Zhang, X.-G.; Yuan, C.-Z.; Gao, B.; Sun, K.; Fu, Q.-B.; Lu, X.-J.; Jiang, J.-C. Acta Phys.-Chim. Sinica 2011, 27, 455 (张海军, 张校刚, 原长洲, 高博, 孙康, 傅清宾, 卢向军, 蒋剑 春, 物理化学学报, 2011, 27, 455.)

[36] Ren, L.; Hui, K. N.; Hui, K. S.; Liu, Y.; Qi, X.; Zhong, J.; Du, Y.; Yang, J. Sci. Rep. 2015, 5, 14229.

[37] Baird, T.; Campbell, K. C.; Holliman, P. J.; Hoyle, R.; Noble, G.; Stirling, D.; Williams, B. P. J. Mater. Chem. 2003, 13, 2341.

[38] Wu, J.; Xu, H.; Zhang, J. Acta Chim. Sinica 2014, 72, 301. (吴娟霞, 徐华, 张锦, 化学学报, 2014, 72, 301.)

[39] Depan, D.; Singh, R. P. J. Appl. Polym. Sci. 2010, 115, 3636.

[40] Xu, Z. P.; Braterman, P. S. J. Mater. Chem. 2003, 13, 268.

[41] Pardieu, E.; Pronkin, S.; Dolci, M.; Dintzer, T.; Pichon, B. P.; Begin, D.; Pham-Huu, C.; Schaaf, P.; Begin-Colin, S.; Boulmedais, F. $J$ Mater. Chem. A 2015, 3, 22877.

\section{Article}

\section{Synthesis and Electrochemical Prop- erties of Nitrogen-Doped Partially Graphitized Carbon/Cobalt Iron Ox- ides Composite}

Li, Tiantian; Zhao, Jikuan*; Li, Yao; Quan, Zhenlan; Xu, Jie Acta Chim. Sinica 2016, 74(X), XXX-XXX

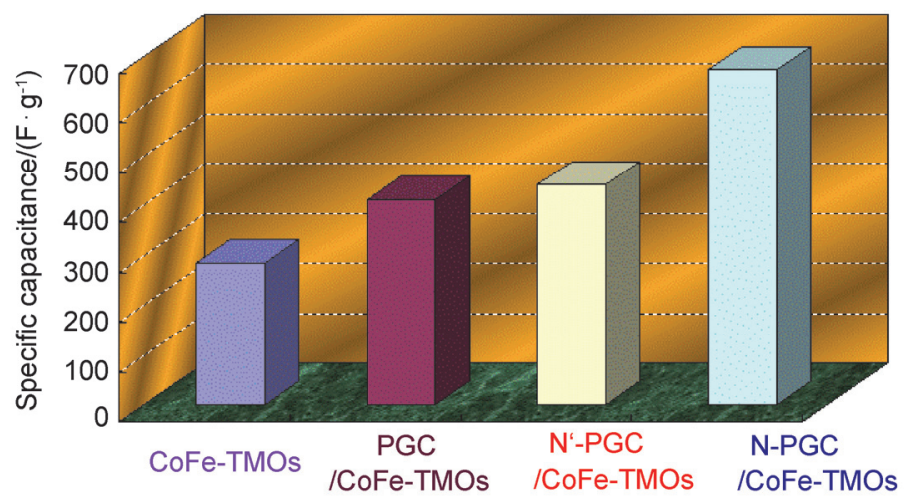

Nitrogen-doped partially graphitized carbon/cobalt iron transition metal oxides nanocomposite (N-PGC/CoFe-TMOs) has been successfully prepared by simple coprecipitation method and calcination treatment, using the biopolymer chitosan as structure directing agent and organic precursor. The $\mathrm{N}-\mathrm{PGC} / \mathrm{CoFe}-\mathrm{TMOs}$ nanocomposite is of superior electrochemical property to CoFe-TMOs (pure oxides), PGC/CoFe-TMOs (composite without nitrogen doping), and N'-PGC/CoFe-TMOs (composite with lower carbon content). 